\title{
Effects of crocin and metformin on methylglyoxal- induced reproductive system dysfunction in diabetic male mice
}

\author{
Maryam Kheirollahi Khorasani ${ }^{1}$, Akram Ahangarpour ${ }^{2}$, Layasadat Khorsandi ${ }^{3}$ \\ 'Department of Physiology, Student Research Committee, ${ }^{2}$ Persian Gulf Physiology Research Center, Medical Basic Sciences Research Institute, and \\ ${ }^{3}$ Department of Anatomical Sciences, Faculty of Medicine, Cellular and Molecular Research Center, Medical Basic Sciences Research Institute, Ahvaz \\ Jundishapur University of Medical Sciences, Ahvaz, Iran
}

Objective: This study investigated the effect of crocin in methylglyoxal (MGO)-induced diabetic male mice.

Methods: Seventy 1-month-old male NMRI mice weighing 20-25 g were divided into seven groups ( $\mathrm{n}=10)$ : sham, MGO (600 mg/kg/day), MGO+crocin (15, 30, and $60 \mathrm{mg} / \mathrm{kg} /$ day), MGO+metformin ( $150 \mathrm{mg} / \mathrm{kg} /$ day), and crocin (60 mg/kg/day). MGO was administered orally for 30 days. Starting on day 14, after confirming hyperglycemia, metformin and crocin were administered orally. On day 31, plasma and tissue samples were prepared for experimental assessments.

Results: Blood glucose and insulin levels in the MGO group were higher than those in the sham group $(p<0.001)$, and decreased in response to metformin $(p<0.001)$ and crocin treatment (not at all doses). Testis width and volume decreased in the MGO mice and improved in the crocin-treated mice $(p<0.05)$, but not in the metformin group. Superoxide dismutase levels decreased in diabetic mice $(p<0.05)$ and malondialdehyde levels increased $(p<0.001)$. Crocin and metformin improved malondialdehyde and superoxide dismutase. Testosterone $(p<0.001)$ and sperm count $(p<0.05)$ decreased in the diabetic mice, and treatment with metformin and crocin recovered these variables. Luteinizing hormone levels increased in diabetic mice $(p<0.001)$ and crocin treatment (but not metformin) attenuated this increase. Seminiferous diameter and height decreased in the diabetic mice and increased in the treatment groups. Vacuoles and ruptures were seen in diabetic testicular tissue, and crocin improved testicular morphology $(p<0.01)$.

Conclusion: MGO increased oxidative stress, reduced sex hormones, and induced histological problems in male reproductive organs. Crocin and metformin improved the reproductive damage caused by MGO-induced diabetes.

Keywords: Crocin; Diabetes mellitus; Methylglyoxal; Oxidative stress; Reproduction

Received: November 21,2020 · Revised: February 23, 2021 · Accepted: March 16, 2021 Corresponding author: Akram Ahangarpour

Department of Physiology, Faculty of Medicine, Persian Gulf Physiology Research Center, Medical Basic Sciences Research Institute, Ahvaz Jundishapur University of Medical Sciences, Ahvaz 61357-15794, Iran

Tel: +98-91-6608-0817 Fax: +98-61-3333-2036

E-mail:akramahangarpour@gmail.com

*This research was extracted from the MSc thesis of Maryam Kheirollahi Khorasani. This study was labeled Project No. APRC-9805. This research was supported financially by the Physiology Research Center, Ahvaz Jundishapur University of Medical Sciences, Ahvaz, Iran.

This is an Open Access article distributed under the terms of the Creative Commons Attribution Non-Commercial License (http://creativecommons.org/licenses/by-nc/4.0/) which permits unrestricted non-commercial use, distribution, and reproduction in any medium, provided the original work is properly cited.

\section{Introduction}

Diabetes poses a serious problem throughout the world, and sexual and reproductive disorders are among the most important secondary complications in patients with diabetes [1]. As the main male reproductive organs of the male, the testes are subjected to various effects of diabetes including altered sperm quality, spermatogenesis, and testicular morphology; changes in Sertoli cell glucose metabolism; decreased Leydig cell number and function; testosterone depletion [2]; and damage of the spermatozoa cell membrane in the seminiferous tubules [3]. Ekhoye et al. [4] stated that testicular oxidative stress has been associated with physiomorphological function in rats' reproductive systems. Other studies have reported reduced 
sperm motility and concentration, erectile dysfunction [5], and decreased fertility in men with diabetes [6]. In people with diabetes, more severe androgen deficiency has been reported than in those without diabetes [7]. Diabetes causes disorders in the precise molecular processes that are essential for sperm quality and function. Various mechanisms can explain diabetes-associated destruction of sperm and damage to the reproductive system, including endocrine disruptions and increased oxidative stress [8].

Methylglyoxal (MGO), the levels of which increase with age and hyperglycemia, is a highly reactive compound and a strong precursor to the creation of advanced glycation end products (AGEs), which are mainly derived from glucose and fructose metabolism and are detoxified by the glycemic system under physiological conditions [9]. Diabetes-induced vascular dysfunction [10] and oxidative stress-induced apoptosis in the vascular endothelium [11] have been linked to the presence of MGO. Since MGO is detoxified through two stages of enzymatic reactions by glyoxalase 1 and 2, using glutathione as a cofactor [12], glyoxalase inducers can be used to prevent and treat possible complications of diabetes [13]. MGO is a toxic byproduct of glycolysis and intracellular stress [14], and it has been noted that MGO can contribute to molecular damage in ovarian dysfunction in the female reproductive system [15].

Metformin treatment is the first line of diabetes mellitus management. Due to the high risk of lactic acidosis [16] and induction of acute kidney injury [17], metformin's future was initially precarious, but the ability of metformin to counter insulin resistance and to reduce hyperglycemia without weight gain or an increased risk of hypoglycemia have enabled it to play a major role in diabetes treatment. Sixty years after its introduction in diabetes treatment, metformin has become the most prescribed glucose-lowering medicine worldwide [16].

Crocin, a water-soluble carotenoid, is an active ingredient of saffron. Pharmacological studies have shown that crocin has anti-inflammatory, anticoagulant, and antitumor activity. Crocin has also been proposed for use as a chemical preservative. Recent studies have shown that crocin exerts antioxidant activity through free radical scavenging [18]. Crocin has also been shown to have a protective effect on sperm parameters and seminiferous tubules in mice under oxidative stress induced by paraquat and in diabetic rats [19].

Diabetes results in increased levels of reactive oxygen species (ROS) and free radicals. ROS include hydroxyl radicals, superoxide anion, hydrogen peroxide, nitric oxide, and lipid peroxidation products such as malondialdehyde (MDA). All ROS are able to react with membrane lipids, nucleic acids, proteins, enzymes, and other small molecules and eventually lead to cellular damage. Antioxidant defense systems include free radical inhibitors including catalase, glutathione peroxidase, and superoxide dismutase (SOD). Whenever the balance between ROS production and antioxidant defense disappears as a result of oxidative stress, various pathological conditions arise that can impair diabetes-related reproductive function [20,21]. Although it has been reported that metformin is the most commonly prescribed glucose-lowering medicine worldwide [16] and crocin is a well-known antioxidant component [18], no study has yet investigated the effects of crocin and metformin on MGO-induced diabetes. Therefore, this study was conducted to evaluate the effects of crocin and metformin on the male reproductive system in an MGO-induced diabetic mouse model.

\section{Methods}

\section{Animals}

The study was performed in 70 1-month-old mice (NMRI) weighing 20-25 g. One week before the study, they were transferred to the animal room and experienced a 12-hour light-dark period with free access to food and water. The mice used in this study were treated in accordance with institutional principles and guidelines on animal care (IR.AJUMS.ABHC.REC.1398.035).

\section{Experimental design}

The mice were randomly divided into seven groups with 10 mice in each group. The experiment lasted for 30 consecutive days. The groups included: group 1, Normal saline administered by gavage; group 2, MGO (600 mg/kg/day) administered by gavage [22]; group $3, \mathrm{MGO}+$ metformin administered at a dose of $150 \mathrm{mg} / \mathrm{kg} /$ day; group 4, MGO+crocin administered at a dose of $15 \mathrm{mg} / \mathrm{kg} /$ day; group 5 , MGO+crocin administered at a dose of $30 \mathrm{mg} / \mathrm{kg} /$ day; group 6 , $\mathrm{MGO}+$ crocin administered at a dose of $60 \mathrm{mg} / \mathrm{kg} /$ day; group 7, crocin administered at an effective gavage dose based on a pilot study.

The duration of diabetes induction was 30 days, and all mice except the sham group received MGO at a dose of $600 \mathrm{mg} / \mathrm{kg} /$ day by gavage for 30 consecutive days. To test the induction of diabetes at the end of each week, the animals' blood glucose was measured using a tail vein blood sample and a glucose level above $180 \mathrm{mg} / \mathrm{dL}$ considered as indicating diabetes [14]. On day 14, after confirming hyperglycemia, metformin and crocin were administered orally. On day 31 of the study, plasma and tissue samples were prepared for experimental assessments. After blood sampling and separation of the epididymis and testis, biochemical parameters were evaluated. Finally, anesthesia was performed 24 hours after the last dose of crocin and metformin with ketamine-xylazine, and after chest dissection, blood samples were taken from the left ventricle and centrifuged. The obtained plasma was stored at $-70^{\circ} \mathrm{C}$ for subsequent experiments, including glucose, insulin, follicle-stimulating hormone (FSH), luteinizing hormone $(\mathrm{LH})$, and testosterone plasma levels, as well as 
oxidative stress parameters including MDA and SOD. For histological examinations, the testes were isolated and stained with Bouin's solution and prepared for histological studies. Cauda epididymis isolation was also performed to measure the sperm count. Plasma levels of insulin, testosterone, $\mathrm{FSH}, \mathrm{LH}$, and oxidative stress-related factors including MDA and SOD were measured using an enzyme-linked immunosorbent assay (ELISA) kit. The sperm count was performed using a hematocytometer. The testes of the animals were quickly fixed in $10 \%$ formalin. After tissue processing, 5- to 7-micron paraffin sections were prepared. Sections were then stained with Bouin's solution, and testicular tissue changes (including testicular weight, width, and length and vacuolation of epithelial cells) were evaluated. The volume of the testes was analyzed using the formula: volume $=\left(D^{2} / 4 \times \pi\right) L \times K$, where $L=$ length, $D=$ width, $K=0.9$, and $\pi=3.14$ [23]. The slides were finally examined by light microscopy.

\section{Sperm assessment}

The cauda epididymis of every mouse was separated and transferred into a petri dish containing normal saline $(0.9 \%)$ and minced into small pieces. The spermatozoa were then vented into surrounding fluid after the pieces were squashed. A drop of petri dish solution was transferred into a Neubauer chamber (depth $0.100 \mathrm{~mm}$ and area $0.0025 \mathrm{~mm}^{2}$ ). The sperm count was assayed manually in white blood cell chambers under light microscopy. Data were expressed as the number of sperm per milliliter [24].

\section{Hormonal and antioxidant assessment}

The plasma levels of insulin, $\mathrm{FSH}, \mathrm{LH}$, and testosterone levels were measured by ELISA kits (DRG Instruments, Marburg, Germany). The hormone detection sensitivity was $1.27 \mathrm{mlU} / \mathrm{mL}$ for $\mathrm{FSH}, 0.856 \mathrm{mlU} / \mathrm{mL}$ for $\mathrm{LH}$, and $0.287 \mathrm{nmol} / \mathrm{L}$ for testosterone. Glucose assessment was performed using biochemical assay kits (Pars Azmoon, Tehran, Iran), with a detection limit of $0.182 \mu \mathrm{lU} / \mathrm{mL}$ in each test tube. SOD (Randox Laboratories, Crumlin, UK) and MDA testing were performed using an ELISA assay kit (ZellBio, Lonsee, Germany). The assay detects the MDA level calorimetrically in a range of $0.78-50 \mu \mathrm{M}$ with $0.1 \mu \mathrm{M}$ sensitivity.

\section{Histopathological assessment}

After blood sampling, the left testes of each mouse separated and fixed in $10 \%$ formalin solution. After tissue processing, 5-to 7-micron paraffin sections were prepared and stained with hematoxylin and eosin. Seven microscopy slides were examined to assess the histological changes (e.g., vacuolation of epithelial cells) in each mouse in all groups. The diameters of the seminiferous tubules and the lumen diameter were measured using Motic Images Plus 3.0 software. The height of the seminiferous epithelium was calculated by subtracting the lumen diameter from the tubules' diameter. For each animal, 100 tubules were analyzed [23].

\section{Statistical assessment}

Data were statistically analyzed using IBM SPSS ver. 25 (IBM Corp., Armonk, NY, USA) as mean \pm standard error of mean. The Kolmogorov-Smirnov test was used to determine the normality of the distribution of the variables in the study. The variance was also tested for homogeneity. One-way analysis of variance and the post hoc least significant difference test were used to determine differences between groups. Differences were considered statistically significant at $p<0.05$.

\section{Results}

\section{Blood glucose, insulin, and antioxidant levels}

Blood glucose levels were higher in the MGO group $(p<0.001)$ than in the sham group and decreased with crocin treatment $(p<0.01)$ and metformin $(p<0.001)$. MGO enhanced insulin levels $(p<0.001)$ in comparison with the sham group, while crocin $(30 \mathrm{mg} /$ $\mathrm{kg} /$ day, $p<0.05)$ and metformin $(p<0.001)$ significantly decreased insulin levels. A crocin dose of $60 \mathrm{mg} / \mathrm{kg} /$ day led to significantly higher insulin levels compared to metformin $(p<0.05)$. The MGO group had lower SOD levels $(p<0.05)$ than the sham group, and SOD levels increased with crocin treatment (MGO+crocin $30 \mathrm{mg} / \mathrm{kg} /$ day and crocin $60 \mathrm{mg} / \mathrm{kg} /$ day, $p<0.05)$ and metformin $(p<0.01)$. The levels of MDA in the MGO group were higher than in the sham group $(p<0.001)$ and decreased with crocin and metformin treatment $(p<0.001)$ (Table 1).

\section{Animal weight and reproductive organ weight}

The animal weight and the reproductive organ weight did not change significantly. Testicular width and testicular volume were significantly lower in the MGO group than in the sham group $(p<0.05)$, and the administration of 30 and $60 \mathrm{mg} / \mathrm{kg}$ of crocin increased these parameters $(p<0.05)$. Metformin treatment did not change these values (Table 2).

\section{Sex hormones and sperm count}

The sperm counts in the MGO and metformin groups were lower than in the sham group $(p<0.05)$ and increased with crocin treatment at all doses in comparison with metformin (Table 3). Testosterone levels were clearly lower in the MGO group than in the sham group $(p<0.001)$ and increased with crocin $(p<0.001)$ and metformin $(p<0.05)$ treatment. Crocin doses of 30 and $60 \mathrm{mg} / \mathrm{kg} /$ day led to significant increases in testosterone levels compared to metformin (Figure 1). LH levels (Figure 2) were significantly higher in the MGO group than in the sham group $(p<0.001)$ and decreased with 
Table 1. Effects of MGO, MT, and crocin on blood glucose, plasma insulin, SOD, and MDA levels in normal and diabetic male mice

\begin{tabular}{|c|c|c|c|c|}
\hline Group & Insulin & Glucose & SOD & MDA \\
\hline Sham & $4.1 \pm 0.1$ & $113.7 \pm 15.8$ & $94.96 \pm 1.42$ & $25.6 \pm 3.3$ \\
\hline MGO & $7.7 \pm 0.6^{c)}$ & $179.3 \pm 15.6^{c)}$ & $81.2 \pm 8.12^{\mathrm{a})}$ & $69.1 \pm 3.4^{c)}$ \\
\hline MGO+MT & $4.3 \pm 0.4^{f)}$ & $127.5 \pm 2.7^{f)}$ & $95.3 \pm 1.5^{\mathrm{e})}$ & $28.6 \pm 9.4^{f)}$ \\
\hline MGO+CR015 & $6.5 \pm 1.6^{c)}$ & $161.5 \pm 15.5^{\mathrm{c})}$ & $92.6 \pm 2.18$ & $52.0 \pm 3.0^{(\mathrm{c}, \mathrm{ff})}$ \\
\hline $\mathrm{MGO}+\mathrm{CRO} 30$ & $5.6 \pm 0.9^{\mathrm{a}), \mathrm{d})}$ & $139.0 \pm 20.0^{\mathrm{e})}$ & $95.5 \pm 1.2^{a)}$ & $38.7 \pm 1.7^{(\mathrm{c}, \mathrm{ff}}$ \\
\hline MGO+CRO60 & $7.2 \pm 0.9^{(\mathrm{c}, \mathrm{g})}$ & $151.0 \pm 3.0^{\mathrm{b}), \mathrm{e})}$ & $92.6 \pm 2.43$ & $40.0 \pm 0.58^{(\mathrm{l}, \mathrm{ff}}$ \\
\hline CRO60 & $6.5 \pm 0.8^{b, g)}$ & $138.2 \pm 11.5^{\mathrm{e})}$ & $93.5 \pm 0.4^{d)}$ & $24.6 \pm 1.3^{\mathrm{f})}$ \\
\hline
\end{tabular}

Values are presented as mean \pm standard error of the mean.

MGO, methylglyoxal; MT, metformin; $\mathrm{SOD}$, superoxide dismutase; $\mathrm{MDA}$, malondialdehyde; $\mathrm{CRO}$, crocin.

a) $p<0.05,{ }^{\text {b) }} p<0.01$, and ${ }^{\text {c) }} p<0.001$ compared to the sham group; ${ }^{\text {d) }} p<0.05$, e) $p<0.01$, and ${ }^{\text {f) }} p<0.001$ compared to the MGO group; ${ }^{\text {g) }} p<0.05$ compared to the MGO+MT group (analysis of variance, followed by the post hoc least significant difference test).

Table 2. Effects of MGO, MT, and crocin on animal weight and male reproductive organs

\begin{tabular}{lccccc}
\hline Group & Body weight $(\mathrm{g})$ & Testis weight $(\mathrm{mg})$ & Testis width $(\mathrm{mm})$ & Testis length $(\mathrm{mm})$ & Testis volume $\left(\mathrm{mm}^{3}\right)$ \\
\hline Sham & $27.0 \pm 0.9$ & $88.2 \pm 6.6$ & $4.8 \pm 0.4$ & $7.5 \pm 0.2$ & $129.7 \pm 27.7$ \\
MGO & $25.02 \pm 0.7$ & $85.75 \pm 2.7$ & $4.0 \pm 0.0^{\text {a) }}$ & $7.03 \pm 0.0$ & $79.41 \pm 3.3^{\mathrm{a})}$ \\
MGO+MT & $28.0 \pm 1.1$ & $91.8 \pm 17.3$ & $4.2 \pm 0.7$ & $7.16 \pm 0.4$ & $90.07 \pm 13.2$ \\
MGO+CRO15 & $23.8 \pm 0.7$ & $89.3 \pm 2.0$ & $4.6 \pm 0.2$ & $7.0 \pm 0.3$ & $105.8 \pm 10.9$ \\
MGO+CRO30 & $26.2 \pm 1.8$ & $85.6 \pm 8.5$ & $5.0 \pm 0.3^{\text {b) }}$ & $7.0 \pm 0.1$ & $126.9 \pm 17.8^{\text {b) }}$ \\
MGO+CRO60 & $25.4 \pm 2.7$ & $96.4 \pm 10.0$ & $5.0 \pm 0^{\text {b) }}$ & $7.5 \pm 0.3$ & $132.5 \pm 5.1^{\text {b) }}$ \\
CRO60 & $26.5 \pm 3.6$ & $98.7 \pm 7.9$ & $5.3 \pm 0.3^{\text {b) }}$ & $7.4 \pm 0.3$ & $131.8 \pm 5.1^{\text {b) }}$ \\
\hline
\end{tabular}

Values are presented as mean \pm standard error of the mean $(n=10)$.

MGO, methylglyoxal; MT, metformin; CRO, crocin.

${ }^{a} p<0.05$ compared to the sham group; ${ }^{\text {b) }} p<0.05$ compared to the MGO group (one-way analysis of variance, followed by the post hoc least significant difference test).

Table 3. Effects of MGO, MT, and crocin on seminiferous morphology and sperm count in normal and diabetic male mice

\begin{tabular}{|c|c|c|c|}
\hline Group & Seminiferous diameter $(\mu \mathrm{m})$ & Seminiferous height ( $\mu \mathrm{m})$ & Sperm count $\left(\times 10^{6}\right) / \mathrm{mL}$ \\
\hline Sham & $272.2 \pm 23.3$ & $159.6 \pm 18.9$ & $3.2 \pm 0.7$ \\
\hline MGO & $211.1 \pm 25.1^{\mathrm{e})}$ & $128.1 \pm 12.6^{\mathrm{a})}$ & $1.4 \pm 0.1^{\mathrm{a})}$ \\
\hline MGO+MT & $271.8 \pm 24.6^{\mathrm{e})}$ & $159.1 \pm 16.4^{d)}$ & $1.7 \pm 0.1^{\mathrm{a})}$ \\
\hline MGO+CRO15 & $253.2 \pm 15.4^{\mathrm{a})}$ & $135.3 \pm 12.8^{\mathrm{a})}$ & $2.7 \pm 0.3^{\mathrm{d})}$ \\
\hline $\mathrm{MGO}+\mathrm{CRO} 30$ & $264.1 \pm 20.2^{d)}$ & $146 \pm 16.6^{\mathrm{d})}$ & $4.4 \pm 0.8^{f(h), h}$ \\
\hline MGO+CRO60 & $223.2 \pm 11.7^{b)}$ & $129.3 \pm 14.2^{\mathrm{a})}$ & $3.1 \pm 0.1^{\mathrm{e}) . \mathrm{g})}$ \\
\hline CRO60 & $273.4 \pm 21.8^{\mathrm{e})}$ & $158.5 \pm 22.5^{\mathrm{d})}$ & $6.5 \pm 0.9^{(c, f), i)}$ \\
\hline
\end{tabular}

Values are presented as mean \pm standard error of the mean.

MGO, methylglyoxal; MT, metformin; CRO, crocin.

a) $p<0.05,{ }^{\text {b) }} p<0.01$, and ${ }^{\text {c) }} p<0.001$ compared to the sham group; ${ }^{\text {d) }} p<0.05,{ }^{\text {e) }} p<0.01$, and ${ }^{\text {f) }} p<0.001$ compared to the MGO group; ${ }^{\text {g) }} p<0.05$, ${ }^{\text {h) }} p<0.01$, and i) $p<0.001$ compared to the MGO+MT group (analysis of variance, followed by the post hoc least significant difference test).

crocin treatment ( 15 and $30 \mathrm{mg} / \mathrm{kg} /$ day $p<0.001$, and $60 \mathrm{mg} / \mathrm{kg} /$ day $p<0.01$ ). Metformin treatment did not change this parameter. FSH levels did not show significant between-group differences (Figure 3).

\section{Testicular histopathology and sperm count}

The observations of testis histology were normal in the sham group. Many vacuoles and epithelial ruptures were observed in the seminiferous tubule epithelium of the MGO-treated diabetic male mice compared to the sham group (Figure 4). The number and size of these vacuoles and damage (Table 4, Figure 4) were lower in the crocin- and metformin-treated diabetic groups (especially at a dose of $30 \mathrm{mg} / \mathrm{kg}$ ). Clear areas of tissue damage such as sloughing of seminiferous epithelium and atrophy were seen in the MGO group $(p<0.001)$, which demonstrated relative improvement in the MGO + crocin group at all doses $(p<0.001)$ and the MGO+metformin group $(p<0.001)$ (Table 4, Figure 4). It has been proven that the diameters 
Table 4. Effects of MGO, MT, and crocin on testis damage in normal and diabetic male mice

\begin{tabular}{|c|c|c|c|c|}
\hline Group & Normal & Sloughing (\%) & Vacuolization (\%) & Atrophy (\%) \\
\hline Sham & $97.1 \pm 0.2$ & $0.7 \pm 0.1$ & $2.2 \pm 0.1$ & 0.00 \\
\hline MGO & $30.4 \pm 4.3^{b)}$ & $11.7 \pm 2.1^{c)}$ & $48.7 \pm 5.4^{c)}$ & $8.2 \pm 1.9^{c)}$ \\
\hline MGO+MT & $96.9 \pm 1.5^{\mathrm{e})}$ & $0.8 \pm 0.2^{\mathrm{e})}$ & $2.3 \pm 0.2^{f)}$ & $0.0 \pm 0.0^{f)}$ \\
\hline MGO+CRO15 & $86.0 \pm 5.7^{\mathrm{d})}$ & $3.3 \pm 0.4^{\mathrm{b}), \mathrm{e})}$ & $9.5 \pm 2.1^{\mathrm{b}), \mathrm{e})}$ & $1.2 \pm 0.2^{(\mathrm{c}, \mathrm{e})}$ \\
\hline $\mathrm{MGO}+\mathrm{CRO} 30$ & $91.2 \pm 5.9^{d)}$ & $0.5 \pm 0.2^{\mathrm{a}), \mathrm{ff}}$ & $6.8 \pm 1.7^{\mathrm{e})}$ & $0.5 \pm 0.1^{b), f)}$ \\
\hline $\mathrm{MGO}+\mathrm{CRO} 60$ & $35.4 \pm 5.2^{b)}$ & $10.7 \pm 2.3^{c)}$ & $46.8 \pm 5.7^{c)}$ & $8.1 \pm 1.7^{c)}$ \\
\hline CRO60 & $97.6 \pm 0.3^{\mathrm{e})}$ & $0.5 \pm 0.2^{f)}$ & $1.9 \pm 0.1^{f)}$ & $0.00^{f)}$ \\
\hline
\end{tabular}

Values are presented as mean \pm standard deviation.

MGO, methylglyoxal; MT, metformin; CRO, crocin.

a) $p<0.05$, b) $p<0.01$, and ${ }^{c)} p<0.001$, compared to the sham group; ${ }^{\text {d) }} p<0.05$, ${ }^{\text {e) }} p<0.01$, and ${ }^{\text {f) }} p<0.001$ compared to the MGO group (analysis of variance, followed by the post hoc least significant difference test).

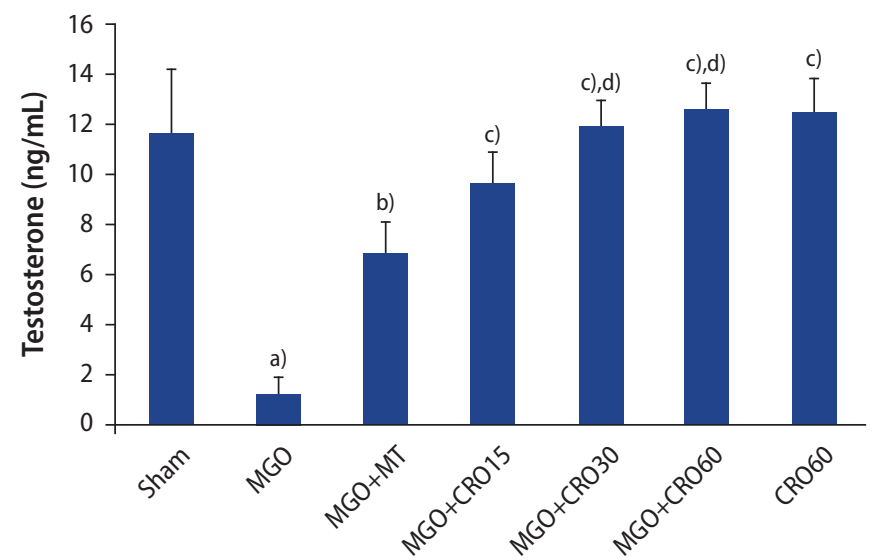

Figure 1. Effects of methylglyoxal (MGO), metformin (MT), and crocin (CRO) on testosterone concentration in all groups. Values are presented as mean \pm standard error $(n=10) .{ }^{a} p<0.001$ compared with the sham group; ${ }^{\text {b) }} p<0.01,{ }^{\mathrm{c}} p<0.001$ compared with the MGO group; ${ }^{\text {d) }} p<0.05$ compared to the MGO + MT group (analysis of variance, followed by the post hoc least significant difference test).

of seminiferous tubules and the epithelium height decreased in diabetic mice compared to the sham group ( $p<0.01$ and $p<0.05$, respectively), and this damage improved in the crocin- and metformin-treated groups (Table 3). Sperm count decreased in the MGO-treated group $(p<0.05)$, while the administration of crocin improved the sperm count in diabetic and non-diabetic groups $(15,30$, and $60 \mathrm{mg} / \mathrm{kg}$ and alone $60 \mathrm{mg} / \mathrm{kg}$ (rocin) in comparison with metformin $(p<0.05)$.

\section{Discussion}

In the present study, the induction of diabetes by MGO was confirmed by measurements of plasma insulin and blood glucose levels. In agreement with this finding, Truong et al. [25] demonstrated that MGO destroyed pancreatic $\beta$-cells by accumulation of AGEs in the

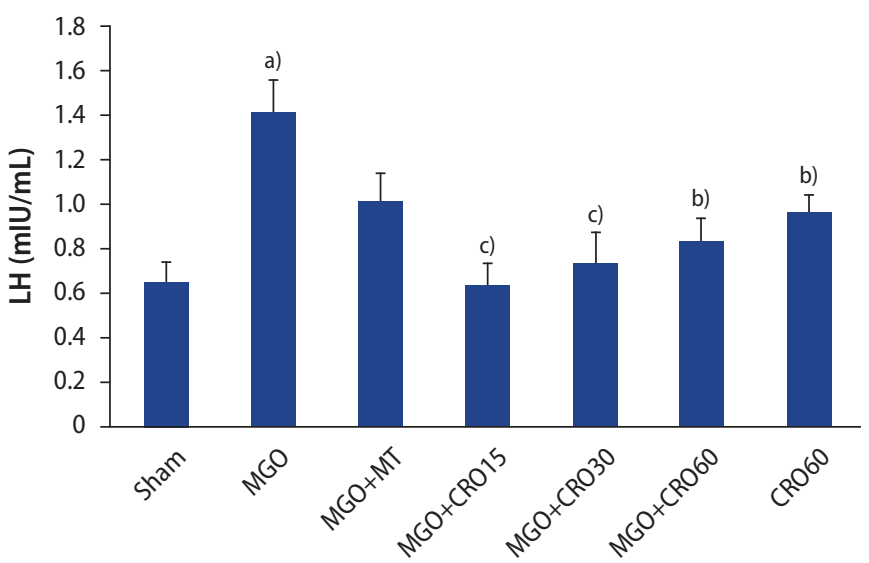

Figure 2. Effects of methylglyoxal (MGO), metformin (MT), and crocin (CRO) on luteinizing hormone $(\mathrm{LH})$ concentrations in all groups. Values are presented as mean \pm standard error $(n=10)$. a) $p<0.001$ compared with the sham group; ${ }^{\text {b) }} p<0.01{ }^{c)} p<0.001$ compared with the MGO group (analysis of variance, followed by the post hoc least significant difference test).

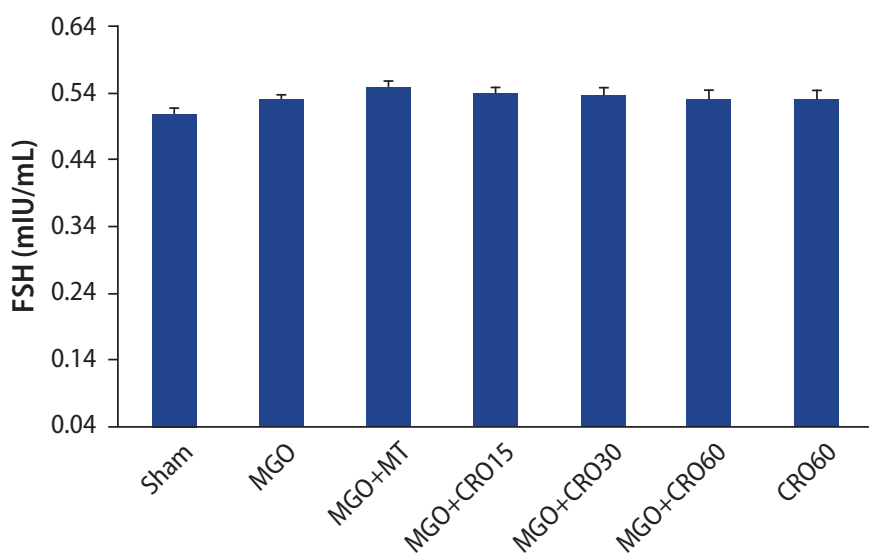

Figure 3. Effects of methylglyoxal (MGO), metformin (MT), and crocin (CRO) on follicle-stimulating hormone (FSH) concentrations in all groups. 


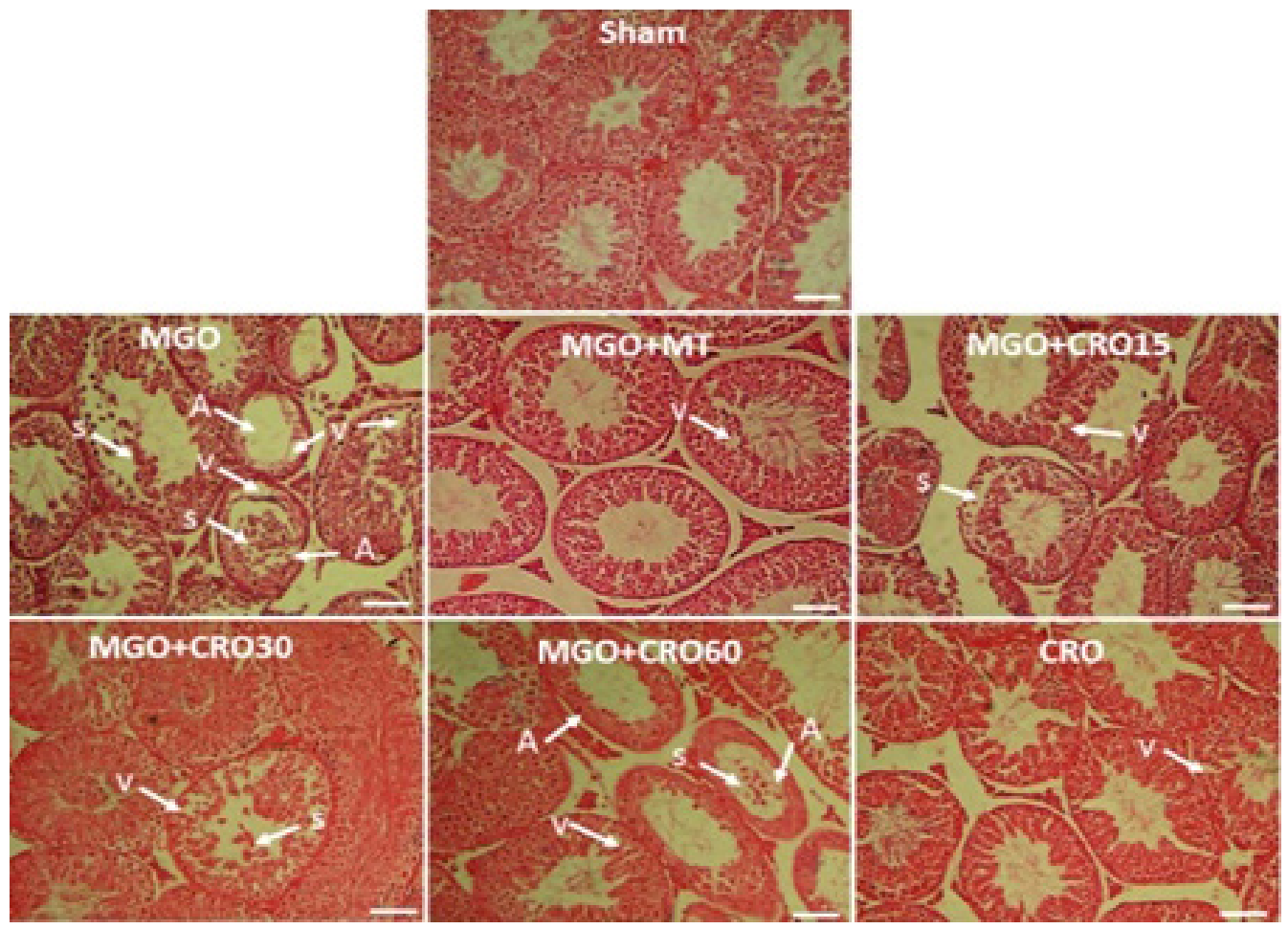

Figure 4. Effects of methylglyoxal (MGO), metformin (MT), and crocin (CRO) on testis histopathology (H\&E). S, sloughing of seminiferous epithelium; $\mathrm{V}$, vacuole; A, atrophy. Scale bar: $100 \mu \mathrm{m}$.

tissue and inflicted insulin resistance. Diabetes induced by MGO changed testis weight, volume, and morphology. High blood glucose levels might cause abnormalities in homeostatic regulation due to changes in glucose consumption by cells. The anterior pituitary gland may be affected by hyperglycemia, resulting in dysfunction of the pituitary-gonadal axis. This phenomenon can destroy germ cells and decrease the size of seminiferous tubules and seminal vesicles [26]. Abnormal glucose homeostasis and hyperglycemia have harmful outcomes on testicular function and spermatogenesis in males with diabetes [7]. Indeed, the findings of the present study revealed that MGO-induced diabetes resulted in male reproductive dysfunction by reducing plasma testosterone levels and sperm count. Furthermore, levels of the antioxidant (SOD) decreased, while those of MDA increased. Diabetic insulin intolerance and hyperglycemia have been associated with dysregulation of the testis, resulting in imbalances of sex steroid hormone levels, such as reduced testosterone secretion by Leydig cells and increased plasma levels of LH. Testosterone secretion from Leydig cells is necessary for spermatogenesis and fertility. This hormone, through its androgen receptors, acts directly on target cells. As a result of stress induced at the testicular lev$\mathrm{el}$, insulin resistance in diabetes is associated with impairments of Leydig cell testosterone secretion and its receptors [27]. In diabetes mellitus, the whole-body metabolism is affected, including endocrine disruption, testicular energy consumption, and male reproduc- tive function. Sertoli cells are protective cells that provide a nutritional supporting environment for somatic and germ cells in the testes, which could be affected by diabetes mellitus. The relationship between male infertility and diabetes has been discussed in some studies based on diabetic models. It has been reported that hyperglycemia leads to increased production of oxidant components like ROS and disruption of antioxidant defenses. Because of excessive ROS generation through hyperglycemia, oxidation of proteins and lipids occurs in the cell, which experiences damage to macromolecules such as protein, lipids, and DNA [28]. In addition, the membranes of spermatozoa are rich in polyunsaturated fatty acids, and this fact makes it susceptible to oxidative stress or damage caused by free radicals [28,29]. Butchi Akondi et al. [28] reported significant reductions in sperm parameters, such as sperm count and motility, in diabetic mice through an increase in oxidative stress and ROS generation. The findings of the present study indicate that diabetes causes oxidative stress, which might be a major mechanism of sperm count reduction; however, further studies are required to determine the exact mechanism.

The present study found that crocin and metformin treatment improved reproductive parameters in diabetic mice. In agreement with these findings, Sefidgar et al. [18] showed in pharmacological studies that crocin had anti-inflammatory, anticoagulant, and anti-tumor activity. Crocin, which is mainly extracted from the saffron plant, has 
also been proposed as a chemical preservative. It has been shown that crocin exerts free radical scavenging activity and therefore has antioxidant activity. Roshankhah et al. [3] also demonstrated that crocin had a protective effect on sperm parameters and seminiferous tubule morphology. Of course, many studies have been investigated to obtain an accurate dose of crocin with the desired antidiabetic effects. Nikbakht-Jam et al. [30] suggested that crocin could behave as both an antioxidant and pro-oxidant, and affects pro-oxidant/anti-oxidant status depending on concentration. The results of this study showed the best effects of crocin at a dose of $30 \mathrm{mg} / \mathrm{kg}$. Interestingly, metformin led to reduced sperm count and testicular volume; the mechanism of action of these side effects of metformin require further study.

In accordance with previous research, the present results indicate that MGO administration changed testis morphology. According to experimental observations, vacuolation in testis tissue is a major symptom of Sertoli cell injury. Consistent with these results, Sertoli cell injury can lead to degeneration of spermatocytes and reduction in sperm count and reproductive factors [31]. Moreover, the role of Sertoli cells is to provide an essential environment for spermatogenesis and germ cell survival. Diabetes mellitus increases the number and size of vacuoles in Sertoli cell cytoplasm in the testes, and decreases seminiferous tubule diameter and epithelium height. All of these changes can affect spermatogenesis [32]. In addition, the present histopathological results showed seminiferous tubule disruption, decreased sperm count, and Sertoli cell dysfunction in the diabetic mice. Any agent resulting in decreased testicular testosterone production or secretion causes narrowing of the seminiferous tubular diameter, luminal epithelium atrophy, or Sertoli cell vacuolization [33]. Based on the results of this study, treatment with crocin and metformin improved diabetes-related and reproductive parameters. However, interestingly, metformin did not improve MGO-induced damage to testicular volume, testosterone secretion, and sperm count as much as crocin. A limitation of this study is the lack of an examination of other sperm parameters such as sperm motility, morphology, and apoptotic changes, and it is suggested that future research should address this gap.

\section{Conflict of interest}

No potential conflict of interest relevant to this article was reported.

\section{ORCID}

Maryam Kheirollahi Khorasani https://orcid.org/0000-0003-0159-0148 Akram Ahangarpour https://orcid.org/0000-0002-9534-9699
Layasadat Khorsandi

https://orcid.org/0000-0002-3391-3055

\section{Author contributions}

Conceptualization: AA. Data curation: AA, MKK. Formal analysis: AA, MKK. Fund acquisition: all authors. Methodology: all authors. Project administration: AA, MKK. Visualization: AA, MKK. Writingoriginal draft: $A A, M K K$. Writing-review \& editing: all authors.

\section{References}

1. Elyasi F, Kashi Z, Tasfieh B, Bahar A, Khademloo M. Sexual dysfunction in women with type 2 diabetes mellitus. Iran J Med Sci 2015;40:206-13.

2. Rabbani N, Thornalley PJ. The critical role of methylglyoxal and glyoxalase 1 in diabetic nephropathy. Diabetes 2014;63:50-2.

3. Roshankhah S, Jalili C, Salahshoor MR. Effects of crocin on sperm parameters and seminiferous tubules in diabetic rats. Adv Biomed Res 2019;8:4.

4. Ekhoye El, Olerimi SE, Ehebha SE. Comparison of the deleterious effects of yaji and cadmium chloride on testicular physiomorphological and oxidative stress status: the gonadoprotective effects of an omega-3 fatty acid. Clin Exp Reprod Med 2020;47:168-79.

5.Karachalias N, Babaei-Jadidi R, Rabbani N, Thornalley PJ. Increased protein damage in renal glomeruli, retina, nerve, plasma and urine and its prevention by thiamine and benfotiamine therapy in a rat model of diabetes. Diabetologia 2010;53:1506-16.

6. Asri-Rezaei S, Tamaddonfard E, Ghasemsoltani-Momtaz B, Erfanparast A, Gholamalipour S. Effects of crocin and zinc chloride on blood levels of zinc and metabolic and oxidative parameters in streptozotocin-induced diabetic rats. Avicenna J Phytomed 2015;5:403-12.

7. Li SY, Zhao YL, Yang YF, Wang X, Nie M, Wu XY, et al. Metabolic Effects of testosterone replacement therapy in patients with type 2 diabetes mellitus or metabolic syndrome: a meta-analysis. Int J Endocrinol 2020;2020:4732021.

8. Ding GL, Liu Y, Liu ME, Pan JX, Guo MX, Sheng JZ, et al. The effects of diabetes on male fertility and epigenetic regulation during spermatogenesis. Asian J Androl 2015;17:948-53.

9. Matafome P, Sena C, Seica R. Methylglyoxal, obesity, and diabetes. Endocrine 2013;43:472-84.

10. Krishnamurthy $P$, Wadhwani A. Antioxidant enzymes and human health. London: IntechOpen; 2012.

11. Mirra P, Nigro C, Prevenzano I, Procopio T, Leone A, Raciti GA, et al. The role of miR-190a in methylglyoxal-induced insulin resistance in endothelial cells. Biochim Biophys Acta Mol Basis Dis 2017; 1863:440-9. 
12. Huang Q, Chen Y, Gong N, Wang YX. Methylglyoxal mediates streptozotocin-induced diabetic neuropathic pain via activation of the peripheral TRPA1 and Nav1.8 channels. Metabolism 2016; 65:463-74.

13. Cardoso S, Carvalho C, Marinho R, Simoes A, Sena CM, Matafome $\mathrm{P}$, et al. Effects of methylglyoxal and pyridoxamine in rat brain mitochondria bioenergetics and oxidative status. J Bioenerg Biomembr 2014;46:347-55.

14. Mojadami S, Ahangarpour A, Mard SA, Khorsandi L. Diabetic nephropathy induced by methylglyoxal: gallic acid regulates kidney microRNAs and glyoxalase1-Nrf2 in male mice. Arch Physiol Biochem 2021;1-8.

15. Tatone C, Carbone MC, Campanella G, Festuccia C, Artini PG, Talesa $V$, et al. Female reproductive dysfunction during ageing: role of methylglyoxal in the formation of advanced glycation endproducts in ovaries of reproductively-aged mice. J Biol Regul Homeost Agents 2010;24:63-72.

16. Bailey CJ. Metformin: historical overview. Diabetologia 2017; 60:1566-76.

17. Arroyo D, Melero R, Panizo N, Goicoechea M, Rodriguez-Benitez P, Vinuesa SG, et al. Metformin-associated acute kidney injury and lactic acidosis. Int J Nephrol 2011;2011:749653.

18. Sefidgar SM, Ahmadi-Hamedani M, Jebelli Javan A, Narenji Sani R, Javaheri Vayghan A. Effect of crocin on biochemical parameters, oxidative/antioxidative profiles, sperm characteristics and testicular histopathology in streptozotocin-induced diabetic rats. Avicenna J Phytomed 2019;9:347-61.

19. Kamali FS, Shahrooz R, Najafi GH, Razi M. Ameliorative effect of crocin on sperm parameters and in vitro fertilization in mice under oxidative stress induced by paraquat. Int J Fertil Steril 2020;13:307-14.

20. Hazman O, Aksoy L, Buyukben A. Effects of crocin on experimental obesity and type-2 diabetes. Turk J Med Sci 2016;46:1593-602.

21. Sapanidou V, Taitzoglou I, Tsakmakidis I, Kourtzelis I, Fletouris D, Theodoridis A, et al. Antioxidant effect of crocin on bovine sperm quality and in vitro fertilization. Theriogenology 2015;84:127382.

22. Hsu WH, Lee BH, Li CH, Hsu YW, Pan TM. Monascin and AITC attenuate methylglyoxal-induced PPARY phosphorylation and degradation through inhibition of the oxidative stress/PKC pathway depending on Nrf2 activation. J Agric Food Chem 2013;61:5996-
6006.

23. Ahangarpour A, Oroojan AA, Aliakbari FR. Effects of C-peptide and nicotinamide on serum LH, FSH, testosterone levels and sperm count in nicotinamide/streptozotocin-induced-diabetes in mice. Acta Endocrinol 2014;10:588-94.

24. Oroojan AA, Ahangarpour A, Paknejad B, Zareian P, Hami Z, Abtahi SR. Effects of myricitrin and solid lipid nanoparticle-containing myricitrin on reproductive system disorders induced by diabetes in male mouse. World J Mens Health 2021;39:147-57.

25. Truong CS, Seo E, Jun HS. Psoralea corylifolia L. Seed extract attenuates methylglyoxal-induced insulin resistance by inhibition of advanced glycation end product formation. Oxid Med Cell Longev 2019;2019:4310319.

26. Aritajat S, Wutteerapol S, Saenphet K. Anti-diabetic effect of Thunbergia laurifolia Linn. aqueous extract. Southeast Asian J Trop Med Public Health 2004;35 Suppl 2:53-8.

27. Liu CY, Hsu YJ, Chien YW, Cha TL, Tsao CW. Dietary resistant maltodextrin ameliorates testicular function and spermatogenesis in streptozotocin-nicotinamide-induced diabetic rats. Andrologia 2016:48:363-73.

28. Butchi Akondi R, Kumar P, Annapurna A, Pujari M. Protective effect of rutin and naringin on sperm quality in streptozotocin (STZ) induced type 1 diabetic rats. Iran J Pharm Res 2011;10:585-96.

29. Khaki A, Khaki AA, Hajhosseini L, Golzar FS, Ainehchi N. The anti-oxidant effects of ginger and cinnamon on spermatogenesis dys-function of diabetes rats. Afr J Tradit Complement Altern Med 2014;11:1-8

30. Nikbakht-Jam I, Khademi M, Nosrati M, Eslami S, Foroutan-Tanha M, Sahebkar A, et al. Effect of crocin extracted from saffron on pro-oxidant-anti-oxidant balance in subjects with metabolic syndrome: a randomized, placebo-controlled clinical trial. Eur J Integr Med 2016;8:307-12

31. Creasy DM. Pathogenesis of male reproductive toxicity. Toxicol Pathol 2001;29:64-76.

32. Trindade AA, Simoes AC, Silva RJ, Macedo CS, Spadella CT. Long term evaluation of morphometric and ultrastructural changes of testes of alloxan-induced diabetic rats. Acta Cir Bras 2013;28:25665.

33. Vidal JD, Whitney KM. Morphologic manifestations of testicular and epididymal toxicity. Spermatogenesis 2014;4:e979099. 